\title{
ANATOMÍA DE LA MADERA DE ESPECIES ARBÓREAS DE UN BOSQUE MESÓFILO DE MONTAÑA: UN ENFOQUE ECOLÓGICO-EVOLUTIVO
}

\author{
Silvia Aguilar-RodríGueZ1 y Josefina Barajas-MORales² \\ ${ }^{1}$ Facultad de Estudios Superiores Iztacala, Universidad Nacional Autónoma de México. \\ Av. de los Barrios No. 1, Los Reyes Iztacala, Tlalnepantla 54090, Edo. de México, México. \\ Correo-e: siagro@campus.iztacala.unam.mx \\ ${ }^{2}$ Instituto de Biología, Universidad Nacional Autónoma de México, Apdo. Postal 70-233, Delegación \\ Coyoacán, México 04510, D.F., México. Correo-e: barajas@ibiologia.unam.mx
}

\begin{abstract}
Resumen: Para conocer las tendencias en anatomía ecológica de la madera de un bosque mesófilo de montaña, se estudiaron 29 especies arbóreas de la región de Ocuilan, Estado de México. Los resultados sugieren que el clima homogéneo de la zona influye en la ausencia de anillos de crecimiento en la mayoría de las especies; al mismo tiempo se observó una relación estrecha entre los anillos y el origen fitogeográfico de los taxa. Con respecto a los demás caracteres anatómicos, existe un elevado porcentaje de maderas con porosidad difusa, elementos de vaso medianos y largos, con diámetros pequeños y placas de perforación escalariforme; fibras largas con paredes delgadas, de tipo libriforme, o fibrotraqueidas con presencia o no de septos; el parénquima axial es escaso y los radios son heterogéneos, de tipo I. Estos caracteres de discuten desde un punto de vista ecológico y evolutivo. Palabras clave: anatomía ecológica, anatomía evolutiva, bosque mesófilo de montaña, Estado de México, madera, Ocuilan.
\end{abstract}

\begin{abstract}
In order to investigate trends in ecological wood anatomy of a cloud forest, 29 tree species from Ocuilan, State of Mexico, were studied. The results suggest that the homogeneous climate of the area determines the absence of growth rings for most species; also, a narrow relationship was observed between rings and the phytogeographic origin of taxa. With respect to the remaining anatomical characters, there is a high percentage of wood with diffuse porosity; also, medium and long vessel elements are common, with small diameters and scalariform perforation plates, along with long fibers with thin walls, of libriform type, or fibrotracheids in which septs may be present or not; axial parenchym is scarce and rays are heterogeneous, of type I. These characters are discussed from an ecological and evolutionary point of view.
\end{abstract}

Key words: cloud forest, ecological anatomy, evolutionary anatomy, wood, Ocuilan, State of Mexico.

$\mathbf{L}$ os bosques mesófilos de montaña en México poseen una elevada riqueza de plantas leñosas, las cuales representan casi la mitad de la flora total reportada para este tipo de vegetación (Rzedowski, 1978, 1996). Sus especies arbóreas poseen una gran variación en fenología, altura de los individuos y arquitectura foliar (Luna-Vega et al., 1999). También se les considera ecosistemas extremadamente diversos y heterogéneos en los aspectos florísticos, taxonómicos y en sus afinidades geográficas (Rzedowski, 1978, 1996; Luna-Vega et al., 1989, 1999, 2001). Como ejemplo de ello en estas áreas se presentan elementos arbóreos de origen boreal, tropical y asiático, mientras que otros se conocen como endémicos de México (Rzedowski, 1996). A pesar de la alta diversidad y la heterogeneidad taxonómica que existe entre los bosques mesófilos de montaña, en nuestro país el estudio anatómico de la madera de sus especies no ha sido interpretado desde una perspectiva ecológico-evolutiva. Investigaciones de este tipo se han realizado en floras regionales de diversas partes del mundo como en Norteamérica (Baas y Carlquist, 1985; Carlquist y Hoekman, 1985; Guthrie, 1989), Europa (Baas y Schweingruber, 1987) y Sudamérica (Lindorf, 1994; Alves y Angyalossy-Alfonso, 2000, 2002); en éstos se sugiere que algunos tipos anatómicos de la madera se ven favorecidos en determinados ambientes físicos. Además, la relación entre factores ambientales y ciertos atributos anatómicos de la madera de material genéticamente diverso ha permitido elaborar hipótesis sobre las estrategias adaptativas y el origen de la diversidad anatómica de la madera en el curso de la evolución (Baas y Miller, 1985). En este sentido, se ha observado que las floras leñosas pueden manifestar en su conjunto uno o varios caracteres que son interpretados 
como retenidos o evolucionados, independientemente de que las especies pertenezcan a un taxón poco o muy especializado (Versteegh, 1968). Para México este tipo de interpretaciones son escasas (Barajas-Morales, 1985) y para los bosque mesófilos de montaña no existen.

A partir del estudio de los caracteres anatómicos de la madera de 29 especies arbóreas de un bosque mesófilo de montaña en México, la presente investigación se ha planteado como propósito dar a conocer algunas tendencias anatómicas de los elementos de la madera, relacionándolas con el clima del área de estudio y con algunos aspectos evolutivos. También se comparan las características anatómicas principales con las de otras comunidades vegetales.

\section{Características de la zona de estudio}

En el Estado de México el bosque mesófilo de montaña representa $3 \%$ de la cobertura vegetal; esta comunidad se desarrolla en la vertiente sur del Eje Neovolcánico y se presenta como pequeñas isletas localizadas principalmente en la parte suroeste de esta entidad. El área de estudio se ubica en las barrancas de Mexicapa, en la ladera sur-sureste del cerro Zempoala (1858' N y 99²0' O), a 40 km al suroeste del centro de la ciudad de México y a $17.5 \mathrm{~km}$ al noroeste de Cuernavaca. En la zona se presenta un clima Cwbg, que corresponde a un templado subhúmedo, el más húmedo de los subhúmedos, con lluvias entre 1,200 y 1,700 mm anuales principalmente en verano y con sólo $5 \%$ del promedio anual en invierno, con una temporada de sequía marcada en invierno-primavera; la temperatura es isotermal, con un promedio anual de $17.5^{\circ} \mathrm{C}$ (Luna-Vega et al., 1989). El bosque mesófilo de montaña en el área se desarrolla entre 1,800 y 2,400 m s.n.m. y se caracteriza fisonómicamente porque sus árboles alcanzan alturas de $25 \mathrm{~m}$, aunque es más común que no pasen de $20 \mathrm{~m}$. Existen árboles de hoja decidua pero nunca se ven desprovistos totalmente de follaje. Se presentan dos estratos arbóreos: uno bajo de 8-12 m y uno alto de 12-25 m, donde los elementos más importantes son Quercus spp., Pinus leiophylla, Ilex tolucana, Carpinus caroliniana y Saurauia reticulata, entre los árboles más altos, y Alnus arguta, Cleyera mexicana, Cornus disciflora, Fucshia arborescens, Meliosma dentata, Styrax ramirezii, Symplocos citrea y Ternstroemia pringlei entre los bajos (Luna-Vega et al., 1989).

\section{Materiales y métodos}

En el campo se seleccionaron 29 especies de árboles maduros y de fustes rectos (cuadro 1), las cuales representan aproximadamente $70 \%$ de las reportadas en el área de estudio (Aguilar-Rodríguez et al., 2000). A una altura de $1.3 \mathrm{~m}$ se obtuvo una rodaja de $5 \mathrm{~cm}$ de grosor que se empleó para realizar los estudios anatómicos y para observar la presencia de anillos de crecimiento. De cada una de las especies se cortaron dos cubos de madera de $2.5 \mathrm{~cm}$ de lado procedentes de las rodajas. Los cubos se ablandaron hirviéndose en agua durante 2 a 5 horas. De ellos se obtuvieron cortes transversales, tangenciales y radiales de 20 a $30 \mu \mathrm{m}$ de grosor, y se tiñeron en una solución acuosa de safranina al 1\%; se sometieron a deshidratación en alcoholes graduales y se aclararon con xilol, para ser montados finalmente en resina sintética. Para el material disociado se obtuvieron astillas de aproximadamente $2 \mathrm{~cm}$ de largo y 1 $\mathrm{cm}$ de grosor y se colocaron en solución de Jeffrey (Johansen, 1940). De este material se tomaron datos como longitud de fibras y elementos de vaso, tipo de fibras, tipo de placas de perforación y número de barras en placas de perforación escalariforme.

Las características microscópicas cualitativas y cuantitativas se designaron de acuerdo con las propuestas de la Asociación Internacional de los Anatomistas de la Madera (IAWA Committee, 1989). Los caracteres microscópicos cualitativos descritos fueron los anillos de crecimiento, tipo de porosidad y el tipo de placa de perforación. También se observaron el tipo de fibras, el parénquima axial (tipo, distribución y abundancia) y composición celular de los radios (Kribs, 1935). De las características microscópicas cuantitativas se tomaron 25 medidas para cada una, obteniéndose la media y la desviación estándar. Estas características fueron longitud de elementos de vaso y diámetro tangencial de los vasos más grandes, incluyendo su pared; de las fibras se midió la longitud, el diámetro tangencial y el grosor de paredes. El grosor de la pared de las fibras fue calificado cualitativamente de acuerdo con las tres categorías indicadas por el comité de la IAWA (1989), como muy delgada, delgada a gruesa y muy gruesa; a esas categorías les asignamos los porcentajes siguientes: < de 25\%, 25 a $75 \%$ $\mathrm{y}>75 \%$, respectivamente. El porcentaje de pared de cada una de las especies estudiadas se obtuvo relacionando el diámetro tangencial de la fibra con el grosor de la pared. La clasificación de las dimensiones de los elementos traqueales se asignó de acuerdo con Chattaway (1932) y la Asociación Internacional de Anatomistas de la Madera (IAWA Committee, 1937, 1939, 1989). Se obtuvo la relación Lf/Lev (longitud de fibra dividida por la longitud de elemento de vaso) para saber el grado de avance filético en los taxa estudiados (Carlquist, 1988).

\section{Resultados}

Los caracteres anatómicos cualitativos y cuantitativos de las maderas estudiadas se muestran en los cuadros 1 y 2 . Descripciones detalladas e ilustradas de cada una de las especies pueden encontrarse en la serie Anatomía de Maderas de México, publicada por el Instituto de Biología de la Universidad Nacional Autónoma de México (AguilarRodríguez et al., 2000). Los resultados muestran algunas tendencias estructurales de la madera de las especies 
Cuadro 1. Características anatómicas cualitativas y cuantitativas de elementos traqueales. $\mathrm{P}=$ porosidad, Diám. $=$ diámetro, Long. $=$ longitud, $\mathrm{Pp}=$ placa de perforación (número de barras), $\mathrm{D}=$ difusa, $\mathrm{A}=$ anular, $\mathrm{SA}=$ semianular, $\mathrm{E}=$ escalariforme, $\mathrm{S}=$ simple, $\mathrm{R}=$ reticulada, $\mathrm{Gp}=$ grosor de pared, $\mathrm{F}=$ fibrotraqueidas, $\mathrm{L}=$ fibras libriformes, $\mathrm{Lf} / \mathrm{Lev}=$ cociente de crecimiento intrusivo.

\begin{tabular}{|c|c|c|c|c|c|c|c|c|c|c|c|}
\hline \multirow{3}{*}{ Especie } & \multicolumn{4}{|c|}{ Elementos de vaso } & \multicolumn{6}{|c|}{ Fibras } & \multirow{3}{*}{$\begin{array}{l}\text { Lf/ } \\
\text { Lev }\end{array}$} \\
\hline & \multirow[t]{2}{*}{$\bar{P}$} & \multirow{2}{*}{$\begin{array}{l}\text { Diám. } \\
(\mu \mathrm{m})\end{array}$} & \multirow{2}{*}{$\begin{array}{l}\text { Long. } \\
(\mu \mathrm{m})\end{array}$} & \multirow[t]{2}{*}{$\mathrm{Pp}$} & \multirow{2}{*}{$\begin{array}{l}\text { Long. } \\
(\mu \mathrm{m})\end{array}$} & \multicolumn{2}{|c|}{ Gp } & \multirow{2}{*}{$\begin{array}{c}\text { Diam. } \\
(\mu \mathrm{m})\end{array}$} & \multirow[t]{2}{*}{ Tipo } & \multirow[t]{2}{*}{$\overline{\text { Septos }}$} & \\
\hline & & & & & & $\overline{(\mu \mathrm{m})}$ & $\%$ & & & & \\
\hline $\begin{array}{l}\text { Alnus acuminata subsp. arguta } \\
\text { (Schltdl.) Furlow }\end{array}$ & $\mathrm{D}$ & $118 \pm 11$ & $792 \pm 148$ & $E(10-16)$ & $1247 \pm 122$ & 2.2 & 12 & $18 \pm 3$ & $\mathrm{~F}, \mathrm{~L}$ & & 1.5 \\
\hline Arbutus xalapensis Kunth & A & $57 \pm 8$ & $534 \pm 90$ & $S / E(1-6)$ & $806 \pm 104$ & 4.8 & 19 & $25 \pm 4$ & $\mathrm{~F}, \mathrm{~L}$ & 1 & 1.5 \\
\hline Arctostaphylos discolor (Hook.) DC. & SA & $61 \pm 8$ & $658 \pm 133$ & $S / E(1-7)$ & $1012 \pm 288$ & 2.3 & 8 & $28 \pm 3.9$ & $\mathrm{~F}, \mathrm{~L}$ & 1 & 1.5 \\
\hline Ardisia compressa Kunth & $\mathrm{D}$ & $101 \pm 14$ & $1596 \pm 152$ & $E(15-31)$ & $3023 \pm 343$ & 5.7 & 16 & $35 \pm 3.3$ & $\mathrm{~L}$ & & 1.8 \\
\hline Buddleja americana L. & $\mathrm{D}$ & $97 \pm 11$ & $485 \pm 65$ & $S$ & $990 \pm 138$ & 4.9 & 19 & $25 \pm 3.5$ & L & & 2.0 \\
\hline Buddleja parviflora Kunth & $\mathrm{D}$ & $92 \pm 12$ & $435 \pm 46$ & S & $977 \pm 76$ & 3.4 & 14 & $24 \pm 3.4$ & L & 1 & 2.2 \\
\hline Carpinus caroliniana Walter & $\mathrm{D}$ & $110 \pm 10$ & $726 \pm 101$ & S & $1572 \pm 180$ & 2.3 & 15 & $15 \pm 2$ & L & & 2.1 \\
\hline Clethra mexicana A.DC. & $\mathrm{D}$ & $85 \pm 8$ & $1435 \pm 240$ & $E(25-35)$ & $2256 \pm 204$ & 6.0 & 18 & $33 \pm 4$ & $\mathrm{~F}$ & & 1.5 \\
\hline Cleyera integrifolia (Benth.) Choisy & $\mathrm{D}$ & $78 \pm 6$ & $1321 \pm 280$ & $\mathrm{E}(30-68)$ & $2320 \pm 351$ & 7.5 & 18 & $40 \pm 2.5$ & $\mathrm{~F}$ & & 1.7 \\
\hline Cornus disciflora Moç. et Sessé ex DC. & $\mathrm{D}$ & $70 \pm 5$ & $1458 \pm 254$ & $\mathrm{E}(21-52)$ & $2201 \pm 190$ & 5.3 & 19 & $27 \pm 4$ & $\mathrm{~F}$ & & 1.5 \\
\hline Crataegus pubescens (Kunth) Steud. & $\mathrm{D}$ & $51 \pm 6$ & $402 \pm 48$ & $S / E(1-3)-R$ & $1186 \pm 178$ & 4.5 & 28 & 16. \pm 4 & $\mathrm{~F}$ & & 2.9 \\
\hline Fraxinus uhdei (Wenzig) Lingelsh. & A & $230 \pm 30$ & $402 \pm 50$ & S & $1409 \pm 165$ & 2.2 & 10 & $22 \pm 2.1$ & L & & 3.5 \\
\hline Fuchsia arborescens Sims & $\mathrm{D}$ & $117 \pm 11$ & $648 \pm 66$ & S & $1011 \pm 105$ & 3.4 & 12 & $28 \pm 3.2$ & L & $2-4$ & 1.5 \\
\hline Garrya laurifolia Hartw. & $\mathrm{D}$ & $66 \pm 6$ & $677 \pm 222$ & $E(4-8)$ & $1450 \pm 222$ & 7.6 & 34 & $22 \pm 2.3$ & $\mathrm{~F}$ & & 2.1 \\
\hline Meliosma dentata (Liebm.) Urban & $\mathrm{D}$ & $134 \pm 12$ & $904 \pm 83$ & $E(4-17)$ & $1523 \pm 180$ & 4.8 & 15 & $31 \pm 3$ & L & & 1.6 \\
\hline Oreopanax peltatus Linden ex Regel & $\mathrm{D}$ & $96 \pm 8$ & $950 \pm 157$ & $S / E(1-7)$ & $1384 \pm 141$ & 4.6 & 16 & $28 \pm 4$ & L & $3-5$ & 1.4 \\
\hline $\begin{array}{l}\text { Oreopanax xalapensis (Kunth) } \\
\text { Decne. et Planch. }\end{array}$ & SA & $110 \pm 11$ & $1142 \pm 93$ & $S / E(3-7)$ & $1665 \pm 240$ & 4.5 & 11 & $38 \pm 3$ & L & $2-5$ & 1.4 \\
\hline Perrottetia longistylis Rose & $\mathrm{D}$ & $75 \pm 5$ & $952 \pm 164$ & $E(25-38)$ & $1565 \pm 164$ & 4.0 & 13 & $30 \pm 4$ & L & $3-6$ & 1.6 \\
\hline Prunus brachybotrya Zucc. & $\mathrm{D}$ & $65 \pm 8$ & $503 \pm 75$ & S & $1487 \pm 94$ & 4.5 & 26 & $17 \pm 2.7$ & L & & 2.9 \\
\hline Rapanea juergensenii Mez & $\mathrm{D}$ & $86 \pm 7$ & $517 \pm 79$ & $S$ & $936 \pm 83$ & 5.7 & 23 & $24 \pm 2.1$ & $\mathrm{~F}$ & & 1.8 \\
\hline Salix bonplandiana Kunth & $\mathrm{D}$ & $121 \pm 9$ & $435 \pm 87$ & $S$ & $992 \pm 106$ & 3.6 & 15 & $24 \pm 3$ & L & & 2.2 \\
\hline Salix paradoxa Kunth & $\mathrm{D}$ & $85 \pm 15$ & $540 \pm 77$ & $S$ & $882 \pm 139$ & 2.0 & 9 & $21 \pm 4$ & L & & 1.6 \\
\hline Saurauia reticulata Rose & $\mathrm{D}$ & $143 \pm 18$ & $1635 \pm 271$ & $\mathrm{E}(12-25)$ & $2876 \pm 351$ & 4.0 & 13 & $30 \pm 4$ & $\mathrm{~F}$ & & 1.7 \\
\hline $\begin{array}{l}\text { Styrax argenteus var. ramirezii } \\
\text { (Greenm.) Gonsoulin }\end{array}$ & $\mathrm{D}$ & $114 \pm 16$ & $1042 \pm 128$ & $E(6-10)$ & $2023 \pm 232$ & 4.1 & 20 & $24 \pm 2.4$ & L & & 1.9 \\
\hline Symplocos citrea Lex. & $\mathrm{D}$ & $92 \pm 9$ & $1655 \pm 182$ & $E(22-40)$ & $2112 \pm 248$ & 6.4 & 17 & $32 \pm 3.3$ & $\mathrm{~F}$ & & 1.2 \\
\hline Ternstroemia pringlei (Rose) Standl. & $\mathrm{D}$ & $85 \pm 8$ & $1101 \pm 183$ & $E(20-38)$ & $1983 \pm 234$ & 6.8 & 21 & $32 \pm 4.2$ & $\mathrm{~F}$ & & 1.8 \\
\hline Tilia houghii Rose & $\mathrm{D}$ & $82 \pm 7$ & $506 \pm 36$ & $S$ & $1472 \pm 158$ & 2.3 & 9 & $24 \pm 3.2$ & L & & 2.9 \\
\hline Xylosma flexuosum (Kunth) Hemsl. & $\mathrm{D}$ & $75 \pm 5$ & $909 \pm 112$ & S & $1551 \pm 230$ & 4.0 & 16 & $25 \pm 3$ & $\mathrm{~L}$ & $1-4$ & 1.7 \\
\hline Zinowiewia concinna Lundell & D & $101 \pm 10$ & $529 \pm 119$ & $\mathrm{~S}$ & $1186 \pm 108$ & 4.8 & 20 & $24 \pm 1.6$ & $\mathrm{~F}$ & & 2.2 \\
\hline
\end{tabular}

arbóreas del bosque mesófilo de montaña de Ocuilan.

Anillos de crecimiento. Un $38 \%$ de las especies muestran anillos de crecimiento, los cuales se definen generalmente por la presencia de hileras continuas de fibras con las paredes gruesas y lúmenes más pequeños en la madera tardía, mientras que el $62 \%$ restante no muestran anillos de crecimiento evidentes. La relación entre los anillos de crecimiento, la fenología foliar y el origen geográfico de los taxa estudiados se muestra en el cuadro 3 .

Vasos. La porosidad se presenta predominantemente difusa (83\%) y sólo es anular en $7 \%$ (Arbutus xalapensis y
Fraxinus uhdei) de las especies. El diámetro promedio de los vasos es menor que $100 \mu \mathrm{m}$ en $62 \%$ de las especies. En todos los taxa los elementos de vaso exhibieron una longitud superior a $400 \mu \mathrm{m}$, que de acuerdo con la clasificación del comité de la IAWA (1939), son medianos (55\%) y largos $(45 \%)$. El $59 \%$ de las especies estudiadas tiene placas de perforación escalariforme con 3 hasta 68 barras (cuadro 1).

Fibras. Las fibras presentan longitudes superiores a $900 \mu \mathrm{m}$ en $94 \%$ de las especies, observándose los valores más altos en Saurauia reticulata y Ardisia compressa, con $2,876 \mu \mathrm{m}$ y $3,023 \mu \mathrm{m}$, respectivamente. De acuerdo con el comité de 
Cuadro 2. Características del parénquima axial y radial. $\mathrm{P}=$ paratraqueal, $\mathrm{A}=$ apotraqueal, $\mathrm{B}=$ bandas; $\mathrm{HE}=$ heterogéneo, $\mathrm{HO}=$ homogéneo, $*$ = Agregados, $+=$ Uniseriados.

\begin{tabular}{|c|c|c|c|c|}
\hline \multirow[b]{2}{*}{ Especie } & \multirow{2}{*}{\multicolumn{2}{|c|}{$\begin{array}{l}\text { Parénquima axial } \\
\text { Distribución y abundancia }\end{array}$}} & \multicolumn{2}{|c|}{ Parénquima radial } \\
\hline & & & Composición & Tipo (Kribs) \\
\hline Alnus acuminata subsp. arguta & $\mathrm{A} / \mathrm{P}$ & Difuso/ escaso & $\mathrm{HO}$ & - \\
\hline Arbutus xalapensis & A & Difuso & $\mathrm{HE}$ & II \\
\hline Arctostaphylos discolor & $\mathrm{A} / \mathrm{P}$ & Difuso/ escaso & $\mathrm{HE}$ & II \\
\hline Ardisia compressa & $\mathrm{A} / \mathrm{P}$ & Difuso en agregados/ escaso & $\mathrm{HE}$ & I \\
\hline Buddleja americana & $\mathrm{A} / \mathrm{P}$ & Muy escaso & $\mathrm{HE}$ & II \\
\hline Buddleja parviflora & $\mathrm{A} / \mathrm{P}$ & Difuso/ escaso & $\mathrm{HE}$ & II \\
\hline Carpinus caroliniana & $\mathrm{A} / \mathrm{P}$ & Difuso escaso y marginal & $\mathrm{HE}$ & I \\
\hline Clethra mexicana & $\mathrm{A} / \mathrm{P}$ & Difuso en agregados/ escaso & $\mathrm{HE}$ & I \\
\hline Cleyera integrifolia & $\mathrm{A} / \mathrm{P}$ & Difuso/ escaso & $\mathrm{HE}$ & I \\
\hline Cornus disciflora & $\mathrm{A} / \mathrm{P}$ & Difuso/ escaso & $\mathrm{HE}$ & 1 \\
\hline Crataegus pubescens & $\mathrm{A} / \mathrm{P}$ & Difuso en agregados/escaso & $\mathrm{HE}$ & III \\
\hline Fraxinus uhdei & $\mathrm{P}$ & Vasicéntrico y aliforme & $\mathrm{HO}$ & - \\
\hline Fuchsia arborescens & $\mathrm{P}$ & Escaso & $\mathrm{HE}$ & I \\
\hline Garrya laurifolia & $\mathrm{A} / \mathrm{P}$ & Difuso en agregados/ escaso & $\mathrm{HE}$ & II \\
\hline Meliosma dentata & $\mathrm{A} / \mathrm{P}$ & Vasicéntrico y confluente & $\mathrm{HE}$ & I \\
\hline Oreopanax peltatus & $\mathrm{A} / \mathrm{P}$ & Difuso/ escaso & $\mathrm{HE}$ & II \\
\hline Oreopanax xalapensis & $\mathrm{A} / \mathrm{P}$ & Difuso/ escaso & $\mathrm{HE}$ & I \\
\hline Perrottetia longistylis & $\mathrm{A} / \mathrm{P}$ & Difuso/ escaso & $\mathrm{HE}$ & I \\
\hline Prunus brachybotrya & $\mathrm{A} / \mathrm{P}$ & Difuso en agregados y marginal & $\mathrm{HE}$ & I \\
\hline Rapanea juergensenii & $\mathrm{P}$ & Escaso & $\mathrm{HE} *$ & II \\
\hline Salix bonplandiana & $P$ & Escaso & $\mathrm{HE}$ & II \\
\hline Salix paradoxa & $\mathrm{P}$ & Escaso & $\mathrm{HE}+$ & - \\
\hline Saurauia reticulata & $\mathrm{A} / \mathrm{P}$ & Difuso en agregados/ escaso & $\mathrm{HE}$ & 1 \\
\hline Styrax argenteus var. ramirezii & $\mathrm{A} / \mathrm{P}$ & Difuso en agregados/ escaso & $\mathrm{HE}$ & I \\
\hline Symplocos citrea & $\mathrm{A} / \mathrm{P}$ & Difuso/ escaso & $\mathrm{HE}$ & I \\
\hline Ternstroemia pringlei & $\mathrm{A} / \mathrm{P}$ & Difuso/ escaso & $\mathrm{HE}$ & 1 \\
\hline Tilia houghii & $\mathrm{A} / \mathrm{P}$ & Difuso en agregados/ escaso & $\mathrm{HO}$ & - \\
\hline Xylosma flexuosum & $\mathrm{A} / \mathrm{P}$ & Muy escaso & $\mathrm{HE}$ & 1 \\
\hline Zinowiewia concinna & $\mathrm{B}$ & Concéntricas de 6-12 células de ancho & $\mathrm{HE}$ & I \\
\hline
\end{tabular}

la IAWA (1937), se clasifican como medianas y largas. El grosor de la pared de las fibras fluctúa entre 8 y $34 \%$, por lo que $90 \%$ de las especies poseen paredes muy delgadas y sólo Crataegus pubescens, Garrya laurifolia y Prunus brachybotrya presentaron un grosor de pared superior a $25 \%$, por lo que se ubican en la categoría de delgadas a gruesas (IAWA Committee, 1989). El 62\% de las especies tiene fibras de tipo libriforme, mientras que $45 \%$ presentan fibrotraqueidas (con punteaduras areoladas); $27 \%$ posee septos. La relación Lf/Lev fue menor que 2 en $65 \%$ las especies (cuadro 1).

Parénquima. El parénquima axial es poco abundante en $69 \%$ de las especies, observándose escaso, pero con una distribución que varía de paratraqueal a apotraqueal difuso y apotraqueal en agregados, y como es común, pueden presentarse combinaciones de éstos en la madera de una sola especie (cuadro 2). El parénquima radial se muestra pre- dominantemente integrado por radios heterogéneos (90\%), con lo márgenes uniseriados largos en 55\% de los taxa; de acuerdo con Kribs (1935), corresponden al tipo I (cuadro 2). Una comparación de los porcentajes de incidencia de los caracteres anatómicos del bosque mesófilo de Ocuilan con los de otras comunidades vegetales similares en el mundo y en México se presenta en el cuadro 4.

\section{Discusión}

Los datos obtenidos de las maderas de Ocuilan mostraron algunas tendencias anatómicas que relacionamos con algunos aspectos climáticos y evolutivos.

La presencia de anillos de crecimiento es un carácter complejo ampliamente estudiado pero aún no totalmente aclarado (Panshin y de Zeeuw, 1970; Metcalfe y Chalk, 1989); ha sido utilizado para la interpretación del clima (Fritts, 1976; Fahn, 1978; Wimmer y Grabner, 2000) y es 
ANATOMÍA DE LA MADERA DE ESPECIES DE BOSQUE MESÓFILO DE MONTAÑA

Cuadro 3. Presencia de anillos de crecimiento, origen geográfico y fenología foliar. Información obtenida de Rzedowski (1978, 1998), Gentry (1982) y J.D. Tejero-Díez (com. pers.).

\begin{tabular}{|c|c|c|}
\hline Especie & Origen & Fenología foliar \\
\hline \multicolumn{3}{|l|}{ Anillos de crecimiento conspicuos } \\
\hline Alnus acuminata subsp. arguta & Boreal & Caducifolio \\
\hline Arbutus xalapensis & Boreal & Perennifolio o subcaducifolio \\
\hline Arctostaphylos discolor & Boreal & Perennifolio o subcaducifolio \\
\hline Carpinus caroliniana & Boreal & Caducifolio o subcaducifolio \\
\hline Crataegus pubescens & Boreal & Caducifolio \\
\hline Fraxinus uhdei & Boreal & Caducifolio \\
\hline Salix bonplandiana & Boreal & Perennifolio o caducifolio \\
\hline Prunus brachybotrya & Boreal & Caducifolio \\
\hline Salix paradoxa & Boreal & Perennifolio o caducifolio \\
\hline Tilia houghii & Boreal & Caducifolio \\
\hline Zinowiewia integerrima & Tropical & Perennifolio o caducifolio \\
\hline \multicolumn{3}{|c|}{ Anillos de crecimiento inconspicuos } \\
\hline Ardisia compressa & Tropical & Perennifolio \\
\hline Buddleja americana & Tropical & Perennifolio \\
\hline Buddleja parviflora & Tropical & Perennifolio \\
\hline Clethra mexicana & Tropical & Subperennifolio \\
\hline Cleyera integrifolia & Tropical & Subperennifolio o caducifolio \\
\hline Cornus disciflora & Boreal & Subcaducifolio o caducifolio \\
\hline Fuchsia arborescens & Tropical & Subperennifolio o caducifolio \\
\hline Garrya laurifolia & Boreal & Perennifolio \\
\hline Meliosma dentata & Tropical & Subperennifolio o caducifolio \\
\hline Oreopanax peltatus & Tropical & Subcaducifolio o perennifolio \\
\hline Oreopanax xalapensis & Tropical & Perennifolio \\
\hline Perrottetia longistilis & Tropical & Caducifolio \\
\hline Rapanea juergensenii & Tropical & Perennifolio \\
\hline Saurauia reticulata & Tropical & Subperennifolio \\
\hline Styrax argenteus var. ramirezii & Tropical & Subperennifolio o caducifolio \\
\hline Symplocos citrea & Tropical & Subperennifolio o caducifolio \\
\hline Ternstroemia pringlei & Tropical & Subperennifolio o caducifolio \\
\hline Xylosma flexuosum & Tropical & Caducifolio \\
\hline
\end{tabular}

afectado por cambios ambientales drásticos como sequías y heladas (Wimmer y Grabner, 2000). En las zonas tropicales húmedas la actividad cambial generalmente es continua a lo largo de todo el año, por lo que la mayoría de las especies pueden no formar anillos de crecimiento evidentes, o desarrollarlos de manera inconspicua, como sucede en climas donde la estacionalidad no es muy marcada (Wheeler et al., 1995). En nuestros resultados, 62\% de las especies estudiadas no mostraron anillos de crecimiento evidentes; este porcentaje parece congruente con la homogeneidad climática presente en el área, pues la temperatura es isotermal y prácticamente no se presentan temperaturas por debajo de $0^{\circ} \mathrm{C}$ (Luna-Vega et al., 1989). Además, la precipitación anual puede ser considerada como intermedia (entre los climas muy húmedos de la selva alta y los secos de los matorrales xerófilos) y la humedad ambiental y del suelo se mantienen relativamente altas, incluso durante los meses más fríos (Rzedowski, 1978). En Brasil, Alves y Angialossy-Alfonso (2000) encontraron resultados similares, pues $52 \%$ del total de las especies que viven en climas mesotérmicos, muy similares al bosque mesófilo de montaña en Ocuilan, no presentaron anillos de crecimiento (cuadro 4).

En las regiones templadas del mundo ha sido más evidente la interrelación entre la presencia de anillos de crecimiento y la fenología foliar (Paliwal y Prasad, 1970), pues en especies caducifolias el desarrollo de hojas nuevas al inicio de la estación de crecimiento demanda un incremento en los tejidos conductores, con la consecuente formación de anillos de crecimiento. En nuestros resultados, tanto las especies con anillos (38\%) como las que no los presentan se comportan indistintamente como caducifolias o como perennifolias (cuadro 3), por lo que las especies de Ocuilan no parecen ajustarse al comportamiento de los árboles de 
Cuadro 4. Porcentajes de características anatómicas en diferentes tipos de vegetación de México y otras regiones del mundo. Bosque Mesófilo, Indonesia, (Versteegh, 1968); Bosque Mesófilo, México (Aguilar-Rodríguez et al., 2000); Selva Baja Caducifolia (Barajas-Morales y LeónGómez, 1989); Bosque Mesotérmico de Brasil (Alves y Angyalossy-Alfonso, 2000, 2002).

\begin{tabular}{|c|c|c|c|c|c|c|}
\hline & & & $\begin{array}{l}\text { Bosque Mesófilo } \\
\text { Ocuilan, México }\end{array}$ & $\begin{array}{l}\text { Bosque Mesófilo } \\
\text { Indonesia }\end{array}$ & $\begin{array}{c}\text { Bosque } \\
\text { Mesotérmico } \\
\text { Brasil }\end{array}$ & $\begin{array}{c}\text { Selva Baja } \\
\text { Caducifolia } \\
\text { México }\end{array}$ \\
\hline \multirow{2}{*}{\multicolumn{2}{|c|}{ Anillos de crecimiento }} & Presentes & 38 & - & 48 & 18 \\
\hline & & Sin anillos & 62 & - & 52 & 82 \\
\hline \multirow{7}{*}{ 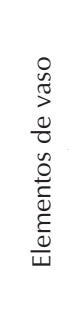 } & \multirow{2}{*}{ Porosidad } & Difusa & 83 & $\longrightarrow$ & 84 & 74 \\
\hline & & Anular & 7 & & -- & 4 \\
\hline & \multicolumn{2}{|c|}{ Diámetro $\mu \mathrm{m}<100$} & 62 & 66 & $\longrightarrow$ & 39 \\
\hline & \multirow{3}{*}{ Longitud $\mu \mathrm{m}$} & Cortos & 0 & - & $\longrightarrow$ & 72 \\
\hline & & Medianos & 55 & - & -- & 27 \\
\hline & & Largos & 45 & - & - & 1 \\
\hline & \multicolumn{2}{|c|}{ Placas Escalariformes } & 59 & 76 & 5 & 0 \\
\hline \multirow{5}{*}{$\frac{\tilde{\pi}}{\frac{\pi}{6}}$} & \multicolumn{2}{|c|}{ Longitud $>900$} & 94 & 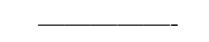 & 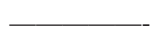 & 68 \\
\hline & Gp & Muy delgadas & 90 & - & 87 & 28 \\
\hline & \multirow{2}{*}{ Tipo } & Libriformes & 62 & 45 & & 96 \\
\hline & & Fibrotraqueidas & 45 & 51 & - & 3 \\
\hline & \multicolumn{2}{|l|}{ Septos } & 24 & - & & 12 \\
\hline \multicolumn{3}{|c|}{$\operatorname{Lf} / \operatorname{Lev}(\mu \mathrm{m})<2$} & 65 & & - & 23 \\
\hline \multicolumn{3}{|c|}{ Parénquima axial escaso } & 69 & 86 & 12 & 12 \\
\hline \multicolumn{3}{|c|}{ Radios Heterogéneos } & 90 & 85 & - & 15 \\
\hline
\end{tabular}

regiones templadas. Por el contrario, coinciden más con las afirmaciones de Tomlinson y Craighead (1972) y Bailey (1980), acerca de que los anillos de crecimiento no siempre están presentes en especies caducifolias o ausentes en las perennifolias. Por otra parte, se hace evidente una importante correlación si se considera el origen fitogeográfico de las especies estudiadas (cuadro 3), ya que $89 \%$ de los taxa que no presentan anillos de crecimiento conspicuos tienen un origen principalmente tropical (Gentry, 1982; Rzedowski, 1978, 1998; Luna-Vega et al., 1989), mientras que los árboles que presentan anillos de crecimiento conspicuos son de origen boreal en $90 \%$ de los casos. Esto coincide con lo que sucede en Eucalyptus camaldulensis (Fhan, 1978), especie que presenta el mismo ritmo de crecimiento independientemente del clima en el que esté creciendo, y su producción de anillos de crecimiento parece ser más bien un carácter endógeno. Por el contrario, encontramos el caso de Garrya y Cornus, ya que siendo ambos géneros de origen boreal, no presentan anillos de crecimiento evidentes, a pesar de que han sido reportados con anillos de crecimiento en otras latitudes (Noshiro y Baas, 1998). Esto indica que la homogeneidad del clima podría haber favorecido la ausencia de anillos de crecimiento en estas dos especies. Todos estos hechos ponen en evidencia la importancia del origen fitogeográfico de las especies y del clima en la presencia de los anillos de crecimiento.

Con respecto a las demás características anatómicas estudiadas, se observa una tendencia a la presencia de porosidad difusa $(83 \%)$, elementos traqueales que se clasifican como medianos y largos (55 y $45 \%$ ) y fibras con paredes muy delgadas (90\%). Estos caracteres pueden estar relacionados con el ambiente húmedo del área, pues en otros trabajos anatómicos las tendencias ecológicas señalan la presencia de porosidad difusa en ambientes en donde no existe una estacionalidad marcada (Woodcock et al., 2000), así como la disminución en la longitud de los elementos traqueales y el incremento de fibras con paredes gruesas conforme aumenta la aridez del clima (Baas, 1983; Baas et al., 1983; Barajas-Morales, 1985, 1987; Lindorf, 1994; Aguilar-Rodríguez et al., 2001), mientras que las especies que disponen de mayor cantidad de humedad o que crecen cerca de corrientes de agua tienen fibras con paredes delgadas (Fahn et al., 1986; Fritts, 1976).

Desde el punto de vista evolutivo, la tendencia en las maderas de Ocuilan a presentar porosidad difusa y elementos de vaso largos, con diámetros pequeños $(<100 \mu \mathrm{m})$ y placas de perforación escalariforme (situación observada en $59 \%$ de las especies), parece haber sido favorecida por el ambiente húmedo de esta zona. Estas características se interpretan como retenidas en especies que no estuvieron sometidas a una presión selectiva para eliminarlas, por el hecho de encontrarse en un clima favorable (Carlquist, 1975; Baas, 1976, 1983).

Carlquist (1988) empleó la relación longitud de fibras/lon- 
gitud de elementos de vaso (Lf/Lev) como indicadora de la cantidad de crecimiento intrusivo y encontró que valores menores de 2 se presentan en familias consideradas como primitivas. Entre las especies estudiadas, $65 \%$ presentó una relación Lf/Lev menor de 2 (cuadros 1 y 4), lo que representa un apoyo más en defensa del carácter menos especializado de la mayoría de las maderas del bosque mesófilo. Otra característica considerada como primitiva o retenida son las fibrotraqueidas (Baas, 1983), que están presentes en $45 \%$ de los taxa trabajados. Este porcentaje es elevado si se compara con las maderas de otros ambientes; las fibrotraqueidas son elementos comunes en zonas montañosas de regiones tropicales climáticamente afines a la zona estudiada (Versteegh, 1968; Barajas-Morales y León-Gómez, 1989; Barajas-Morales et al., 1997; Abundiz-Bonilla et al., 2004).

El parénquima axial es escaso en $69 \%$ de las especies de Ocuilan y dicha escasez es congruente con la presencia de fibras septadas, pues éstas sustituyen al parénquima en su función de almacén (Carlquist, 1988). En términos evolutivos, Wheeler y Baas (1991) interpretan al parénquima axial escaso como un carácter primitivo que, junto con los radios multiseriados heterogéneos, fueron comunes en las primeras angiospermas. Los radios heterogéneos con los márgenes uniseriados largos tipo I (Kribs, 1935) fueron frecuentes en este estudio; según Kribs (1935) estos radios, además del parénquima axial difuso, elementos de vaso con gran longitud y placas de perforación escalariforme, caracterizan a maderas primitivas (Carlquist, 1988). Estos caracteres se presentan en un alto porcentaje de las especies estudiadas y también se hallan en proporciones similares en los bosques mesófilos de Indonesia y los mesotérmicos de Brasil, a diferencia de su escasa presencia en las maderas de la selva baja caducifolia (cuadro 4) y en otros tipos de vegetación de México (Barajas-Morales y León-Gómez, 1989; Barajas-Morales et al., 1997; Abundiz-Bonilla et al., 2004).

En conclusión, el origen fitogeográfico de los taxa estudiados mostró una fuerte relación con la presencia o ausencia de los anillos de crecimiento, ya que éstos están presentes principalmente en los géneros de origen boreal, pero al mismo tiempo el clima homogéneo de Ocuilan parece favorecer la ausencia de dichos anillos en la mayoría de las especies. Por el contrario, la relación de anillos de crecimiento con la fenología foliar no es clara.

En este estudio se corrobora la tendencia comúnmente aceptada de que en los ambientes húmedos existe un elevado porcentaje de maderas con elementos traqueales medianos y largos, porosidad difusa y fibras con pared delgada. Las placas de perforación escalariformes en elementos de vaso largos, presencia de fibrotraqueidas, cocientes Lf/Lev menores que 2, parénquima axial escaso y radios heterogéneos con sus márgenes uniseriados largos, se interpretan en este estudio como caracteres retenidos, opinión que se apoya en las afirmaciones de Baas (1976), Carlquist (1975, 1988) y Metcalfe y Chalk (1989), para maderas actuales y en las de Wheeler y Baas (1991), para maderas fósiles. Desde un punto de vista exclusivamente florístico, Rzedowski (1998) menciona que los bosques mesófilos de montaña actuales en México son relictuales y que sus especies han tenido una larga historia evolutiva; esta afirmación coincide con el hecho de que las maderas del bosque mesófilo de montaña de Ocuilan presentan varios caracteres anatómicos que han sido considerados como filogenéticamente retenidos.

\section{Literatura citada}

Abundiz-Bonilla L.A.M., Barajas-Morales J. y Tenorio-Lezama P. 2004. Anatomía de Maderas de México: Árboles y Arbustos del Matorral Xerófilo de Tehuacán, Puebla. Instituto de Biología, Universidad Nacional Autónoma de México, México, D.F.

Aguilar-Rodríguez S., Abundiz-Bonilla L. y Barajas-Morales J. 2001. Comparación de la gravedad específica y características anatómicas de la madera de dos comunidades vegetales en México. Anales del Instituto de Biología, Universidad Nacional Autónoma de México, Serie Botánica 72:171-185.

Aguilar-Rodríguez S., Barajas-Morales J. y Tejero-Díez J.D. 2000. Anatomía de Maderas de México: Especies de un Bosque Mesófilo de Montaña. Instituto de Biología, Universidad Nacional Autónoma de México, México, D.F.

Alves E.S. y Angyalossy-Alfonso V. 2000. Ecological trends in the wood anatomy of some Brazilian species. 1. Growth rings and vessels. International Association of Wood Anatomists Journal 21:3-30.

Alves E.S. y Angyalossy-Alfonso V. 2002. Ecological trends in the wood anatomy of some Brazilian species. 2. Axial parenchyma, rays and fibres. International Association of Wood Anatomists Journal 23:391-418.

Baas P. 1976. Some functional and adaptative aspects of vessel member morphology. En: Baas P., Bolton A.J. y Catling D.M. Eds. Wood Structure in Biological and Technological Research. pp. 157-181, Leiden University Press, Leiden.

Baas P. 1983. Ecological patterns in xylem anatomy. En: Givnish T.J. Ed. On the Economy of Plant Form and Function, pp. 327352, Cambridge University Press, Cambridge.

Baas P. y Carlquist S. 1985. A comparison of the ecological wood anatomy of the floras of Southern California and Israel. International Association of Wood Anatomists Bulletin new series 6:349-353.

Baas P. y Miller S. 1985. Functional and ecological wood anatomy: Some introductory comments. International Association of Wood Anatomists Bulletin new series 6:281-282.

Baas P. y Schweingruber F.H. 1987. Ecological trends in the wood anatomy of trees, shrubs and climbers from Europe. International Association of Wood Anatomists Bulletin new series 8:245-274.

Baas P., Werker E. y Fahn A. 1983. Some ecological trends in vessel characters. International Association of Wood Anatomists Bulletin new series 4:141-159.

Bailey D.C. 1980. Anomalous growth and vegetative anatomy of Simmondsia chinensis. American Journal of Botany 67:147-161. 


\section{Silvia Aguilar-RodrígueZ y Josefina Barajas-Morales}

Barajas-Morales J. 1985. Wood structural differences between trees of two tropical forests in Mexico. International Association of Wood Anatomists Bulletin new series 6:355364.

Barajas-Morales J. 1987. Wood specific gravity in species from two tropical forests in Mexico. International Association of Wood Anatomists Bulletin new series 8:143-148.

Barajas-Morales J., Ángeles-Álvarez G. y Solís-Sánchez P. 1997. Anatomía de Maderas de México: Especies de una Selva Alta Perennifolia. Instituto de Biología. Universidad Nacional Autónoma de México. México, D.F.

Barajas-Morales J. y León-Gómez C. 1989. Anatomía de Maderas de México: Especies de una Selva Baja Caducifolia. Instituto de Biología. Universidad Nacional Autónoma de México. México, D.F.

Carlquist S. 1975. Ecological Strategies of Xylem Evolution. California University Press, Berkeley.

Carlquist S. 1988. Comparative Wood Anatomy. Springer-Verlag, Berlín.

Carlquist S. y Hoekman D.A. 1985. Ecological wood anatomy of the woody southern Californian flora. International Association of Wood Anatomists Bulletin new series 6:319347.

Chattaway M.M. 1932. Proposed standards for numerical valves used in describing woods. Tropical Woods 29:20-28.

Fahn A. 1978. Anatomía Vegetal. H. Blume Ediciones, Madrid.

Fahn A., Werker E. y Baas P. 1986. Wood Anatomy and Identification of Trees and Shrubs from Israel and Adjacent Regions. The Israel Academic of Science and Humanities, Jerusalén.

Fritts H.C. 1976. Tree Rings and Climate. Academic Press, Nueva York.

Gentry A.H. 1982. Neotropical floristic diversity: Phytogeographical connections between Central and South America, Pleistocene climatic fluctuations, or accident of the Andean orogeny? Annals of the Missouri Botanical Garden 69:557-593.

Guthrie L.R. 1989. Xylem structure and ecological dominance in a forest community. American Journal of Botany 76:12161228.

IAWA Committee. 1937. Committee on the standardization of terms of cell size. Standard terms of lengths of vessel members and wood fiber. Tropical Woods 51:21-22.

IAWA Committee. 1939. Committee on the nomenclature standard terms of size for vessel diameter and ray width. Tropical Woods 59:51-52.

IAWA Committee. 1989. IAWA list of microscopic features for hardwood identification. International Association of Wood Anatomists Bulletin new series 10:219-332.

Johansen D.A. 1940. Plant Microtechnique. McGraw-Hill, Nueva York.

Kribs A.D. 1935. Salient lines of structural specialization in the wood rays in dicotyledons. Botanical Gazette 96:547-557.

Lindorf H. 1994. Eco-anatomical wood features of species from a very dry tropical forest. International Association of Wood Anatomists Journal 15:361-376.

Luna-Vega I., Alcántara-Ayala O., Espinosa-Organista D. y Morrone J.J. 1999. Historical relationships of the Mexican cloud forests: a preliminary vicariance model applying Parsimony Analysis of Endemicity to vascular plant taxa.
Journal of Biogeography 26:1299-1305.

Luna-Vega I., Almeida-Leñero L. y Llorente-Bousquets J. 1989. Florística y aspectos fitogeográficos del bosque mesófilo de montaña de las cañadas de Ocuilan, Estados de Morelos y México. Anales del Instituto de Biología, Universidad Nacional Autónoma de México, Serie Botánica 59:63-87.

Luna-Vega I., Morrone J.J., Alcántara-Ayala O. y EspinosaOrganista D. 2001. Biogeographical affinities among Neotropical cloud forests. Plant Systematics and Evolution 228:229-239.

Metcalfe C.R. y Chalk L. 1989. Anatomy of the Dicotyledons. Vol II. 2a. ed. Clarendon Press, Oxford.

Noshiro S. y Bass P. 1998. Systematic wood anatomy of Cornaceae and allies. International Association of Wood Anatomists Bulletin new series 19:43-97.

Paliwal G.S. y Prasad N.U.S.R.K. 1970. The cambium of Alseuosmia. Phytomorphology 9:5-8.

Panshin A.J. y De Zeeuw C. 1970. Textbook of Wood Technology. Vol. I. McGraw-Hill, Nueva York.

Rzedowski J. 1978. Vegetación de México. Limusa, México, D.F.

Rzedowski J. 1996. Análisis preliminar de la flora vascular de los bosques mesófilos de montaña de México. Acta Botanica Mexicana 35:25-40.

Rzedowski J. 1998. Diversidad y orígenes de la flora fanerogámica de México. En: Ramamoorthy T.P., Bye R., Lot A. y Fa J. Eds. Diversidad Biológica de México: Orígenes y Distribución, pp. 129-145, Universidad Nacional Autónoma de México, México, D.F.

Tomlinson P.B. y Craighead F.C. 1972. Growth-ring studies on the native trees of sub-tropical Florida. En: Ghouse A.K.M. Ed. Research Trends in Plant Anatomy, pp. 39-51, K.A. Chowdhury Commemoration Volume. Tata McGraw-Hill, Nueva Delhi.

Versteegh C. 1968. An anatomical study of some woody plants of mountain flora in the tropics (Indonesia). Acta Botanica Neerlandica 17:151-159.

Wheeler A.E. y Baas P. 1991. A survey of the fossil record for dicotyledonous wood and its significance for evolutionary and ecological wood anatomy. International Association of Wood Anatomists Bulletin new series 12:275-332.

Wheeler A.E., McClammer J. y La Pasha A.C. 1995. Similarities and differences in dicotyledonous woods of the Cretaceous and Paleocene. San Juan Basin, New Mexico, USA. International Association of Wood Anatomists Journal 16:223254.

Wimmer R. y Grabner M. 2000. A comparison of tree-ring features in Picea abies as correlated with climate. International Association of Wood Anatomists Journal 21:403-416.

Woodcock W.D., Dos Santos G. y Reynel C. 2000. Wood characteristics of Amazon forest types. International Association of Wood Anatomists Journal 21:277-292.

Fecha de recepción: 18 de agosto de 2004

Versión corregida: 27 de junio de 2005

Aceptado: 5 de octubre de 2005 